\title{
HeRO monitoring to reduce mortality in NICU patients
}

\author{
This article was published in the following Dove Press journal: \\ Research and Reports in Neonatology \\ 14 August 2012 \\ Number of times this article has been viewed
}

\author{
Karen D Fairchild' \\ Judy L Aschner ${ }^{2}$ \\ 'Department of Pediatrics, University \\ of Virginia School of Medicine, \\ Charlottesville,VA, ${ }^{2}$ Department \\ of Pediatrics, Vanderbilt University \\ School of Medicine, Nashville, TN, \\ USA
}

\begin{abstract}
In 2011, the results of a large, multicenter, randomized clinical trial of heart rate characteristics (HeRO) monitoring in preterm infants were published. Remarkably, in approximately 3000 very low birthweight (VLBW) patients in nine neonatal intensive care units (NICUs) in the US, randomization to having the HeRO score displayed to clinicians resulted in a greater than $20 \%$ reduction in mortality compared with infants whose HeRO score was not displayed. In this trial, sponsored by the National Institutes of Health, the number needed to "treat" or monitor was 48 for each additional VLBW survivor. For extremely low birthweight infants, the number needed to monitor was 23 for each additional survivor. The HeRO score incorporates two heart rate components, decreased variability and decelerations, that occur in a variety of pathologic conditions, most notably sepsis. The HeRO score was designed as an early warning system for sepsis in NICU patients. In the clinical trial, mortality among patients with blood culture-positive sepsis dropped from $16 \%$ in controls to $10 \%$ in those whose HeRO scores were displayed. Ongoing analyses are investigating whether the HeRO score is also a useful clinical or research tool for identifying other neonatal pathologies, such as necrotizing enterocolitis and acute brain injury. The purpose of this review is four-fold: (1) to provide clinicians with a background on the physiology of heart rate regulation in health and disease, (2) to describe how HeRO monitoring was developed, (3) to review results of the randomized clinical trial, and (4) to discuss use of the HeRO monitor for early detection of potentially catastrophic illness in preterm infants in the NICU.
\end{abstract}

Keywords: heart rate variability, neonatal sepsis, preterm infant mortality

\section{Improving survival in preterm infants: evidence-based approaches}

Survival of very low birthweight (VLBW) preterm infants has improved substantially in the past two decades as clinicians have adopted evidence-based therapies, the most significant of which were antenatal corticosteroids and postnatal surfactant. Nonetheless, mortality among VLBW infants remains high at about $10 \%$ and is inversely associated with gestational age. ${ }^{1}$ Sepsis is the leading cause of death in infants surviving beyond their first week in the neonatal intensive care unit (NICU). ${ }^{2}$ Late-onset sepsis in NICU patients can be indolent or explosive, with mortality ranging from less than $10 \%$ to greater than $30 \%$ depending on host vulnerability and on pathogen virulence. The toll of neonatal sepsis also extends to survivors, who have longer hospital stay and worse neurodevelopmental outcome compared with age-matched preterm infants without sepsis. ${ }^{3}$
Correspondence: Karen D Fairchild Department of Pediatrics, Box 800386, University of Virginia Health System, Hospital Drive, Charlottesville,

VA 22908, USA

Tel +l 4349245496

Fax +I 4349828347

Email kdf2n@virginia.edu 
Strategies to reduce morbidity and mortality due to sepsis in hospitalized preterm infants can be divided into prevention, early detection, and adjunctive treatments (beyond antibiotics). A variety of immunomodulatory agents have been tested but have not proven beneficial for either prevention or treatment of neonatal sepsis. The best preventative strategy is hand and line hygiene, but despite the best efforts of clinicians, approximately $25 \%$ of VLBW infants and $50 \%$ of extremely low birth weight (ELBW) infants experience a bloodstream infection in the course of their NICU stay. Early detection of sepsis, prior to overt clinical deterioration, would allow earlier institution of antibiotics and supportive therapies, likely resulting in better outcomes. The difficulty with early detection lies in the subtle and nonspecific nature of clinical signs in the early phase of neonatal sepsis. Administering antibiotics to every preterm infant with subtle signs consistent with sepsis, such as apnea or feeding intolerance (which occur commonly in preterm infants without infection) is not a sound or evidence-based practice, and mounting evidence demonstrates multiple adverse effects of antibiotic overuse. Three strategies under investigation for early detection of sepsis are biomarker screening, rapid molecular diagnostics, and physiomarker monitoring. Extensive research into sepsis biomarkers has yielded insights into the systemic inflammatory response but has not yet translated into clinically useful diagnostic tests for ruling sepsis in or out. Progress on the molecular diagnostics front has been slowed by difficulties in rapid and reliable detection of pathogens in small blood volumes. Another problem with biomarker screening and molecular diagnostics is that blood tests are typically obtained once a patient has signs of sepsis. Physiomarker screening, in the form of computerized vital sign analysis, has the advantage of being available continuously, thus potentially alerting clinicians when a patient in the intensive care unit (ICU) is in the early transition from a "well" to "ill" state, before onset of recognizable clinical signs or symptoms.

Development of the HeRO (heart rate observation) monitor was prompted by the observation that physiologic perturbations in the early phase of sepsis are reflected by subtle complex changes in heart rate characteristics. Decreased heart rate variability and transient repetitive heart rate decelerations, similar to those seen in fetuses in the setting of asphyxia, occur in neonatal sepsis, often before a patient displays obvious clinical signs (Figure 1). These changes are not apparent to clinicians using traditional cardiorespiratory monitoring. The HeRO monitor displays a number or score, which represents the risk that a patient will experience a clinical deterioration consistent with clinical or cultureproven sepsis in the next 24 hours. ${ }^{4}$

\section{Computerized vital sign pattern analysis: early warning systems for deterioration}

Before focusing specifically on heart rate analysis, we will consider more generally how computer analysis of vital sign patterns could be used to improve outcomes of ICU patients. Current state-of-the-art bedside ICU monitors display vital signs in real time (a snapshot) and store and display vital sign trends over time (multiple snapshots). Two things are lacking, analysis of changes in vital sign patterns associated with impending clinical deterioration and integration of multiple vital signs into the analysis. Pathophysiologic conditions, such as sepsis, acute brain injury, or acute respiratory failure, are associated with changes in heart rate, respiration, blood pressure, and temperature, some of which are independent and others interdependent. The complexity of these interactions and the multitude of data presented to busy or less experienced clinicians make it difficult, if not impossible, to predict and intervene before onset of clinical and possibly life-threatening deterioration.

Computer analysis integrating multiple vital signs has been attempted to a limited extent. For adult ICU patients, for example, computer software was developed that analyzes cardiac and respiratory monitor data (electrocardiogram, end-tidal $\mathrm{CO}_{2}$, chest impedance, and oxygen saturation) and this system is undergoing testing to monitor severity of illness and predict sepsis and extubation failure. ${ }^{5}$ For NICU patients, researchers involved in developing the HeRO monitor have developed a new computerized system to analyze respiratory, oxygen saturation, and electrocardiogram waveform data to quantitate apnea, ${ }^{6,7}$ but whether integration of respiratory and heart rate pattern analysis will prove more efficacious than heart rate characteristics monitoring alone for early detection of life-threatening conditions is currently unknown.

\section{Terminology: HRV versus HRC}

Depressed heart rate variability (HRV) refers to a paucity of normal small accelerations and decelerations of heart rate and is indicative of various pathologies. Presence of repetitive transient decelerations (larger than the physiologic dips in heart rate contributing to normal HRV but often not large enough to trigger bradycardia alarms on bedside monitors) may also be pathologic. Heart rate characteristics (HRC) of decreased HRV and transient decelerations are both incorporated into the HRC index, or HeRO score. In this review, 


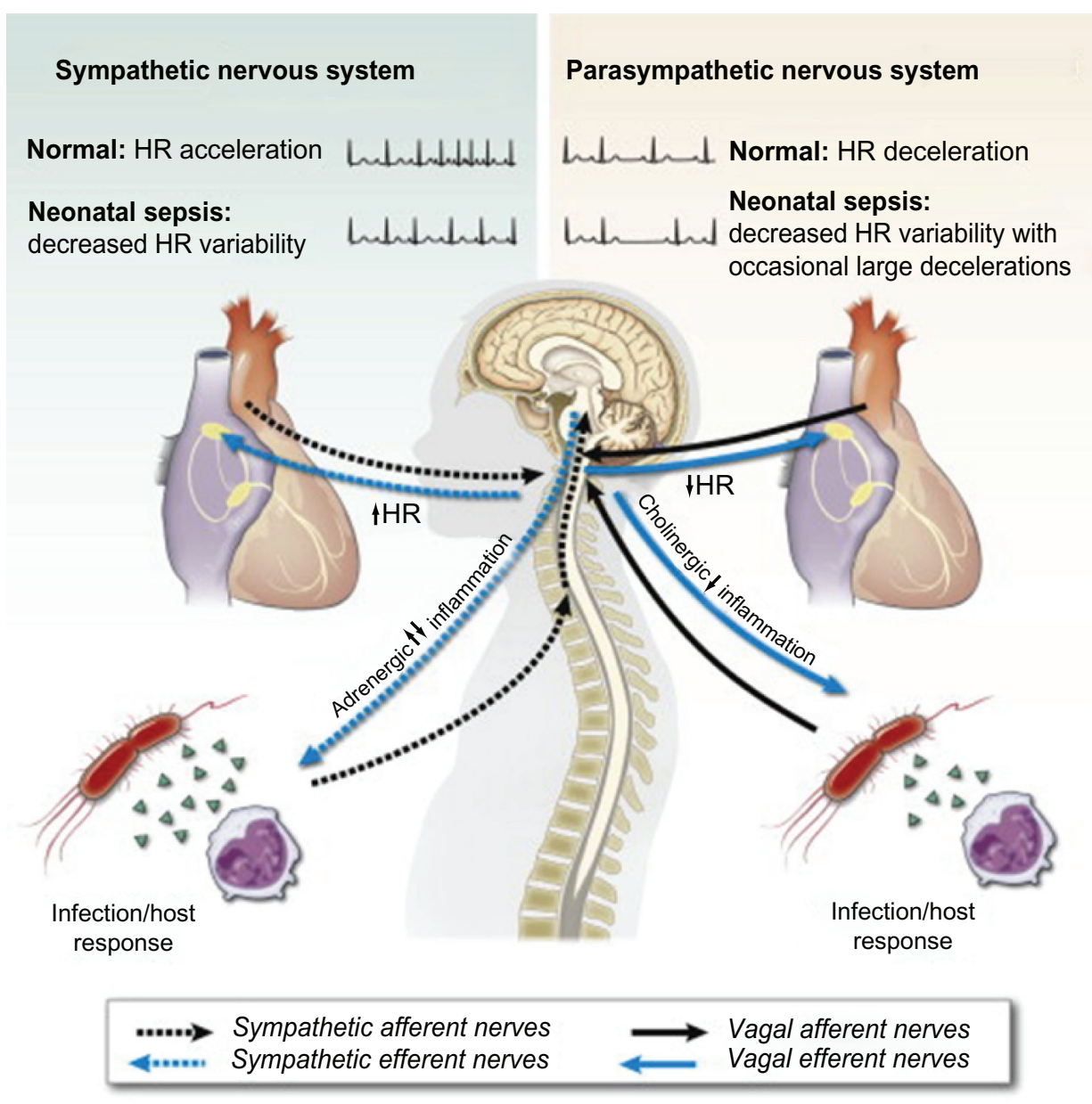

Figure I Effects of autonomic nervous system activation on heart rate characteristics and host response in sepsis.

Notes: In the healthy state, sympathetic activation leads to release of norepinephrine, which increases heart rate, and parasympathetic (vagus nerve) activation leads to release of acetylcholine, which decreases heart rate. Pathogens and inflammatory cytokines can activate the autonomic nervous system, which can in turn alter the inflammatory cytokine cascade. Sepsis can lead to altered sensitivity to adrenergic and cholinergic stimuli (fewer heart rate accelerations and exaggerated decelerations).

the term HRV will be used in discussions of pathophysiology and the terms HRC index or HeRO score in discussions of clinical applications.

\section{Physiology of heart rate regulation in health and disease}

Heart rate is regulated, for the most part, by the autonomic nervous system. Sinoatrial node pacemaker cells in the right atrium respond to neurotransmitters released in response to sympathetic or parasympathetic nerve firing. With sympathetic activation, norepinephrine is released which increases heart rate, and with parasympathetic (vagus nerve) activation, acetylcholine is released, which decreases heart rate. Accelerations and decelerations of heart rate thus reflect the autonomic nervous system balance which changes in response to physiologic needs. HRV is influenced by blood pressure, respiration, and temperature. Through the baroreflex, alterations in blood pressure lead to feedback changes in sympathetic and parasympathetic tone, leading to compensatory changes in heart rate and blood pressure. For example, an acute increase in blood pressure is sensed by baroreceptors leading to decreased sympathetic and increased parasympathetic activity, with a consequent decrease in both blood pressure and heart rate. Respiration affects heart rate through physiologic phenomena referred to as respiratory sinus arrhythmia and cardiorespiratory coupling. Respiratory sinus arrhythmia is a normal physiologic response whereby inspiration is associated with increased heart rate and expiration with decreased heart rate, via alterations in sympathetic and parasympathetic activity. Cardiorespiratory coupling refers to heart beats consistently occurring at the same phase of the ventilatory cycle. ${ }^{7}$ Body temperature also impacts heart rate (with hypothermia leading to sinus bradycardia and hyperthermia to tachycardia), and has a minor impact on HRV.

HRV is depressed in a number of acute and chronic pathologic conditions in adults, including myocardial infarction, 
congestive heart failure, diabetes, and renal failure. ${ }^{8}$ Acute brain injury and psychiatric disorders, such as major depression, are also associated with depressed HRV. ${ }^{9,10}$ In many of these conditions, HRV has been used as a marker of disease severity and as a tool for gauging response to therapies.

Acute uteroplacental insufficiency leading to fetal asphyxia is an example of abnormal HRC well known to obstetricians and neonatologists. In this condition, hypoxia and acidosis are manifest in the fetal heart rate tracing by decreased beat-to-beat variability and repetitive transient decelerations. These abnormal fetal heart rate patterns have also been described in the setting of chorioamnionitis. In the most severe cases, there is a sinusoidal pattern in the fetal heart rate, with repetitive, regular increases and decreases in heart rate of 5-15 beats per minute every 10-30 seconds, a pattern associated with severe fetal anemia or severe hypoxia and an ominous prognosis. ${ }^{11}$

Sepsis leads to decreased HRV in adults, children, and neonates. ${ }^{4,12,13}$ Inflammatory cytokines released as part of the systemic inflammatory response to infection have been found to be associated with decreased HRV in both preclinical and clinical studies. Sepsis-associated downregulation of sympathetic and parasympathetic responses via alterations in adrenergic and cholinergic receptor number, ligand binding, and downstream signal transduction have also been described. ${ }^{14}$

\section{Brain-heart-immune interactions in sepsis}

Changes in heart rate and its variability in sepsis reflect, in large part, direct or indirect effects on autonomic nervous system activation (Figure 1). Tachycardia may reflect increased sympathetic drive via release of endogenous catecholamines, or it may be a compensatory response to intravascular volume depletion due to capillary leak from cytokine damage to endothelial cells. Tachycardia itself can slightly decrease HRV in healthy states, but in sepsis, sympathetic overactivation can eventually lead to decreased responsiveness of adrenergic receptors and a decrease in the normal accelerations of beating rate. Another component of abnormal HRC in neonates with sepsis is repetitive transient decelerations, which appear to reflect, at least in part, parasympathetic (vagus nerve) activation. The vagus nerve plays a key role in host defense against pathogens via the "cholinergic antiinflammatory pathway". ${ }^{15}$ In this response, vagus nerve firing leads to dampening of inflammatory cytokine production by leukocytes, via nicotinic cholinergic receptors. Rodent studies have shown that electrical stimulation of the vagus nerve or administration of nicotine receptor agonists reduces cytokine levels and improves survival in sepsis. ${ }^{16}$ Heart rate decelerations occurring in sepsis may reflect endogenous activation of this critical host defense pathway.

\section{HRV measurement: analyses based on time versus frequency}

The simplest and most widely used measure of HRV is the standard deviation of normal interbeat (RR) intervals, or SDNN (standard deviation of "normal", meaning that ectopic beats and artifacts are removed). Frequency-based analysis of $\mathrm{HRV}$ is also used, which takes into account the occurrence of high and low frequency oscillations in heart rate which reflect autonomic nervous system balance. To perform this type of analysis, time series data (RR intervals) are transformed to determine variability (calculated as "power") within different frequencies. ${ }^{17}$ In adults, high frequency HRV refers to regular accelerations and decelerations that occur from $0.15-0.4 \mathrm{~Hz}$, which corresponds to the normal respiratory rate (9-24 breaths per minute). In neonates, the frequency spectrum for high-frequency HRV is higher, in the range of $0.4-1 \mathrm{~Hz}$, corresponding to their faster respiratory rate of 24-60 breaths per minute. This normal pattern, called respiratory sinus arrhythmia, reflects vagus nerve activity, which decreases with inhalation and increases with exhalation. In contrast with a very rapid response to vagus nerve activation resulting in heart rate slowing, the response to sympathetic stimulation is much slower. This is reflected by low frequency HRV occurring in adults in the range of $0.04-0.15 \mathrm{~Hz}$ (2-9 cycles per minute), which is regulated by sympathetic or a combination of sympathetic and parasympathetic tone. Frequency-based or power spectrum analysis of HRV is commonly used in clinical research involving older patients as a measure of autonomic nervous system balance. In neonates, a number of factors make frequency-based HRV analysis problematic, including high respiratory rates. In neonates with sepsis, our group has shown that frequency-based HRV analysis does not provide additional information over time-based analysis, because both high and low frequency HRV are depressed with no change in the LF:HF ratio. ${ }^{18}$

\section{Development of the HeRO monitoring system}

The HeRO monitor was originally developed as an early warning system for sepsis in preterm infants in the NICU. Researchers at the University of Virginia observed that NICU patients often had abnormal HRC (decreased HRV and transient decelerations) prior to and at the time of clinical 
presentation of sepsis. ${ }^{19}$ Using electrocardiographic data from over 300 NICU patients with over 100 episodes of sepsis, investigators devised an algorithm to quantitate these pathologic changes. ${ }^{20}$ The HRC index incorporates three components, standard deviation of the RR intervals, sample asymmetry, and sample entropy. ${ }^{21,22}$ Sample asymmetry refers to the relative frequency of accelerations and decelerations of heart rate. Presence of repetitive transient decelerations, together with paucity of accelerations, leads to asymmetry and is abnormal. Sample entropy measures irregularity and can be interpreted in the light of modern ideas about uncoupling of organ signaling in systemic inflammatory response syndrome. ${ }^{23}$ The final algorithm is based on logistic regression adjusted for repeated measures, and its result is the probability of sepsis in the next 24 hours. This is normalized by the average probability of sepsis, and clinicians are presented with the fold-increase in risk of imminent sepsis, the HRC index, or HeRO score. A HeRO score of 1 is "normal" for healthy VLBW infants, and a score of 5 indicates a five-fold increased risk that the patient will be diagnosed with sepsis within the next 24 hours.

After the HeRO score was developed at the University of Virginia, it was validated for sepsis detection in over 300 NICU patients at Wake Forest University. ${ }^{20}$ In a subsequent analysis of over 1000 NICU patients at the two centers, it was shown that an elevated HeRO score was associated with sepsis and with mortality. ${ }^{24}$

\section{Technical aspects of HeRO monitoring}

The HeRO monitor has had 510(k) clearance from the United States Food and Drug Administration since 2003 for monitoring HRV and decelerations. It extracts electrocardiographic data from existing NICU monitors; no additional sensor or other hardware is in direct contact with the baby. The HeRO score, which is calculated in real-time and displayed hourly, represents HRC over the previous 12 hours. The monitor display can include views of HeRO scores for all NICU patients, for those in a geographic area of the NICU, or for individual patients (Figure 2). The individual patient display shows the HeRO trend over the past five days, the heart rate trend over the past 30 minutes, the real-time electrocardiogram, and the current HeRO score (Figures 3 and 4).

\section{Randomized clinical trial of HeRO monitoring in VLBW infants}

In order to determine the clinical utility of HeRO monitoring in NICU patients, a randomized clinical trial was carried out in nine NICUs across the US. VLBW infants, stratified by birthweight of $<1000$ grams or 1001-1500 grams, were randomized to having their HeRO score displayed to clinicians or having their HeRO score measured but not displayed. Clinicians were educated about the HeRO score, but no protocol-mandated intervention was required for patients with an increase in HeRO score. Patients were followed for 120 days after randomization or until death or NICU discharge. The primary outcome was days alive and not on mechanical ventilation, and four secondary outcomes were analyzed, ie, mortality, duration of mechanical ventilation, days on antibiotics, and duration of hospitalization. A total of 3003 patients were randomized, and 2989 patients completed the study, making this the largest randomized clinical trial of VLBW infants published to date.

Regarding the primary outcome, patients whose HeRO scores were displayed had a mean of 2.3 more days alive and not on mechanical ventilation compared with those whose HeRO score was not displayed, but this difference was not statistically significant $(P=0.08)$. Importantly, mortality was $22 \%$ lower in the HeRO display group compared with the HeRO nondisplay group $(10.2 \%$ versus $8.1 \%, P=0.04$, Figure 5). In a prespecified subgroup analysis of ELBW infants, the relative reduction in death was $26 \%$ in HeRO displayed versus HeRO nondisplayed groups $(P=0.02)$. The number of ELBW infants needed to monitor with HeRO for one additional life saved was 23 .
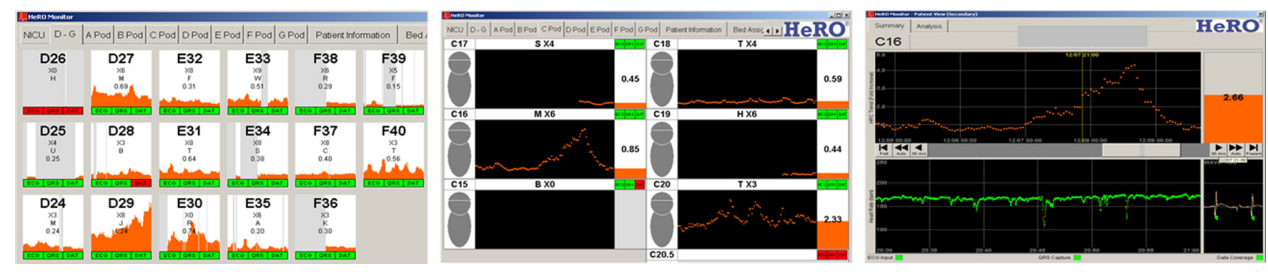

Figure 2 HeRO monitor screens.

Notes: Three displays of the heart rate characteristics index (HeRO score). Left: unit view shows 5-day HeRO score trends (orange) for multiple patients. Center: pod view shows 5-day HeRO score trends (orange line) and current HeRO score in patients from a particular area of the neonatal intensive care unit. Right: individual patient view (see also Figure 3). Abbreviation: HeRO, heart rate observation. 


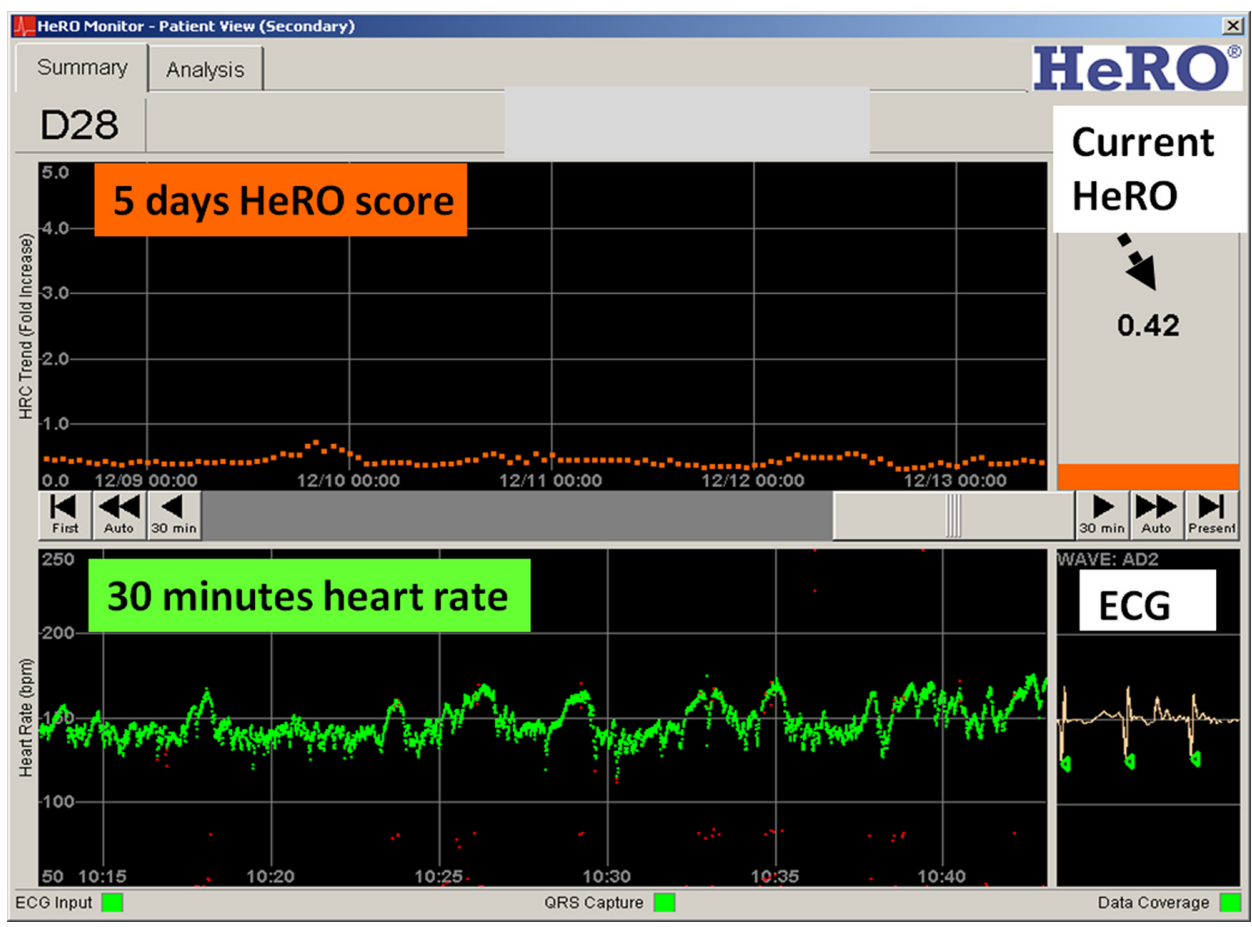

Figure 3 HeRO monitor showing normal heart rate characteristics.

Notes: The top graph (orange line) shows the 5-day trend in the heart rate characteristics index (HeRO score). The HeRO score is calculated continuously from the heart rate variability and transient decelerations, and is the fold increase in risk that the patient will experience a clinical deterioration consistent with sepsis in the next 24 hours. The bottom graph shows the last 30 minutes of heart rate (beats per minute), showing normal accelerations and normal decelerations. At the right is the current HeRO score (0.42, indicating a low chance of imminent sepsis) and the electrocardiogram.

Abbreviation: HeRO, heart rate observation.

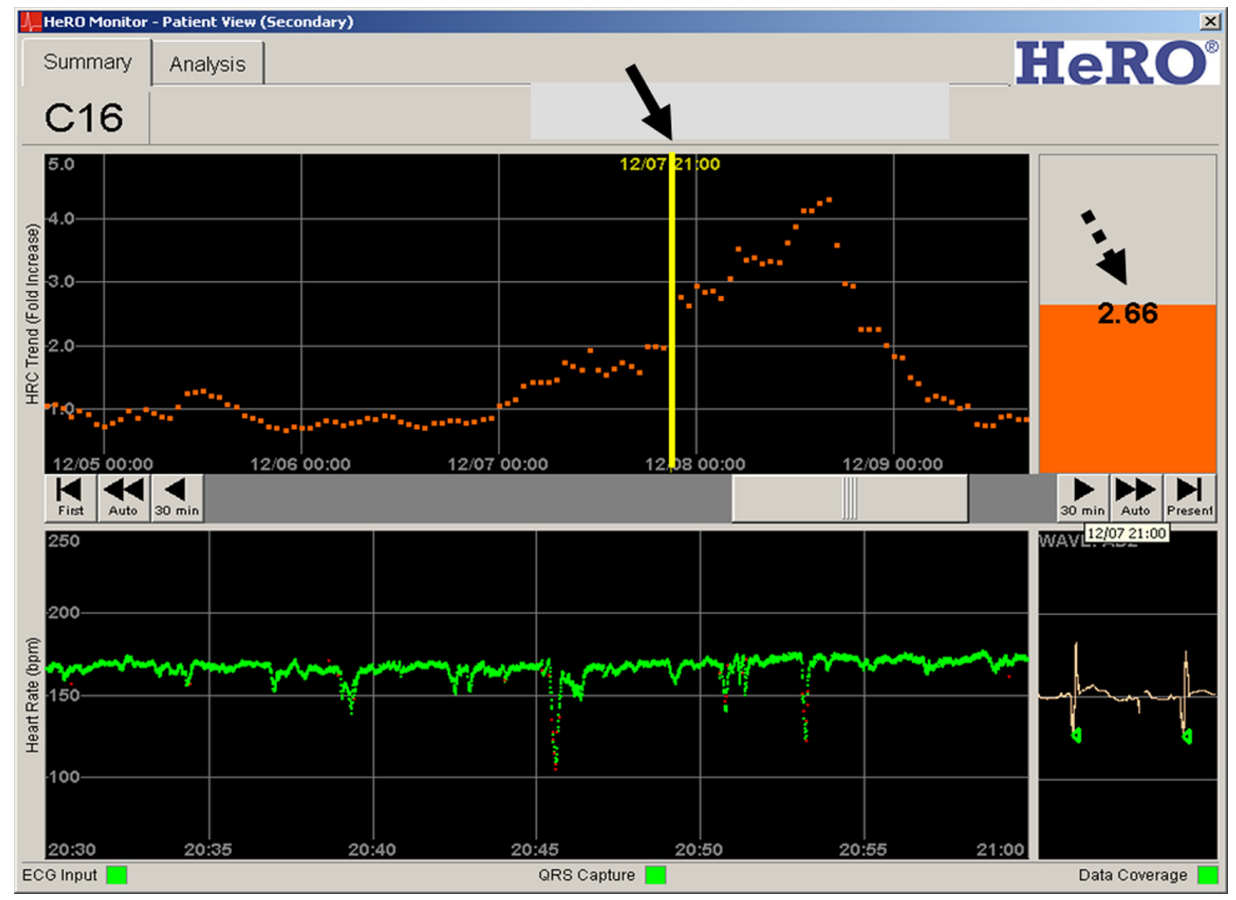

Figure 4 HeRO monitor showing abnormal heart rate characteristics.

Notes: The top graph (orange line) shows two days of low-risk HeRO score (approximately I) followed by a gradual increase in the score over 36 hours, peaking at 4 (indicating four-fold increased risk that the patient will have a clinical deterioration consistent with sepsis in the next 24 hours). At the time indicated by the yellow line and arrow, the HeRO score is 2.66 . The 30-minute heart rate tracing at that time (green, lower panel) shows a baseline heart rate of 165 beats per minute with few accelerations (decreased variability) and superimposed repetitive transient decelerations.

Abbreviation: HeRO, heart rate observation. 


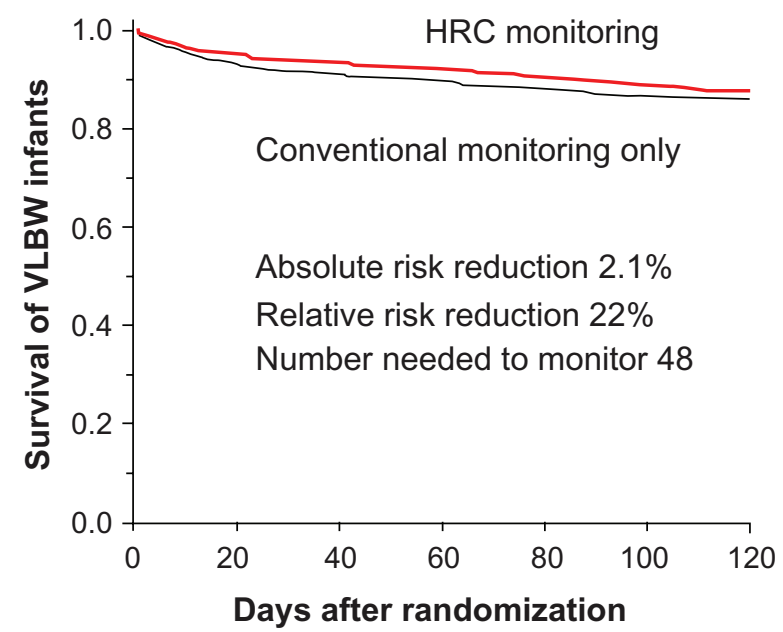

Figure 5 Mortality reduction in HeRO monitor trial, in which 3003 very low birthweight infants were randomized to have their HeRO score displayed to clinicians (HRC monitoring) or stored but not displayed (conventional monitoring).

Note: There was a $22 \%$ relative reduction in mortality $(P=0.04)$ in the HeRO display group.'

Abbreviation: HeRO, heart rate observation.

In the clinical trial, the incidence of blood cultureproven sepsis was not different in the two study groups ( $24 \%$ and $25 \%$ ) but the 30 -day mortality after the first episode of sepsis was lower in the HeRO display versus the HeRO masked group ( $10.0 \%$ versus $16.1 \%$ mortality, $P=0.01$ ). Two important questions regarding HeRO monitoring were whether it increases the number of sepsis evaluations and whether more antibiotics were given when the HeRO score was displayed. In the clinical trial, a slightly higher number of blood cultures were drawn for suspicion of sepsis in the HeRO displayed group (1.8 versus 1.6 blood cultures per month, $P=0.05)$. There was also a slightly higher, but statistically insignificant, number of days on antibiotics in the HeRO displayed group (15.7 versus $15.0, P=0.31$ ).

\section{Practical aspects of HeRO monitor use}

Optimum use of the HeRO monitor for improving patient outcomes in the NICU hinges on two things, a system for alerting the medical team when the score abruptly increases, and an understanding of how to interpret HeRO scores in individual patients. The monitor does not have alarms, so it is up to clinicians to devise a system for being aware of acute increases in the HeRO score. For example, bedside nurses can document the HeRO score with other vital signs and alert other caregivers if there is an increase of more than one point in the HeRO score over the patient's baseline. Generally, a score of 1 or less is "normal" (low risk for sepsis), and in studies of thousands of preterm infants, more than $70 \%$ of the
HeRO scores fall in this low range, whereas fewer than $10 \%$ of HeRO scores fall in the "high risk" range of $>2 .{ }^{24}$

There is no specific threshold HeRO score above which a certain course of action is recommended for individual patients. Rather, the score and the trends in the score should be incorporated into the overall clinical assessment. A patient with significant clinical signs of sepsis should be started on antibiotics regardless of HeRO score or any other test results. On the other end of the spectrum, for the high-risk preterm infant with an abrupt, significant increase in HeRO in the absence of clinical signs of sepsis, initiation of antibiotics rather than waiting for clinical deterioration may be in the patient's best interests. The middle ground, and most common scenario, is the patient with mild, subtle, and/or nonspecific clinical signs that might or might not be attributable to sepsis. In this case, adjunct tests such as a HeRO score or blood tests can help in clinical decision-making about whether to obtain cultures and start antibiotics. A practical "score card" for decision-making based on both clinical signs and HeRO score was devised and tested (Figure 6). ${ }^{25}$ Points were given for clinical signs of sepsis (2 points for severe apnea, feeding intolerance, or immature to total neutrophil ratio $>0.2$; 1 point for increased ventilatory support, lethargy or hypotonia, temperature instability, hyperglycemia, or abnormal white blood cell count). In a study of 337 VLBW infants, a clinical score of 2 or more or a HeRO score in the high-risk range $(>3)$ was associated with a 3-4-fold increased risk of sepsis, whereas a low-risk HeRO and clinical score was associated with a low risk of sepsis.

The HeRO score by itself has been shown to have comparable or better predictive accuracy for sepsis compared with laboratory tests such as blood glucose, white blood cell

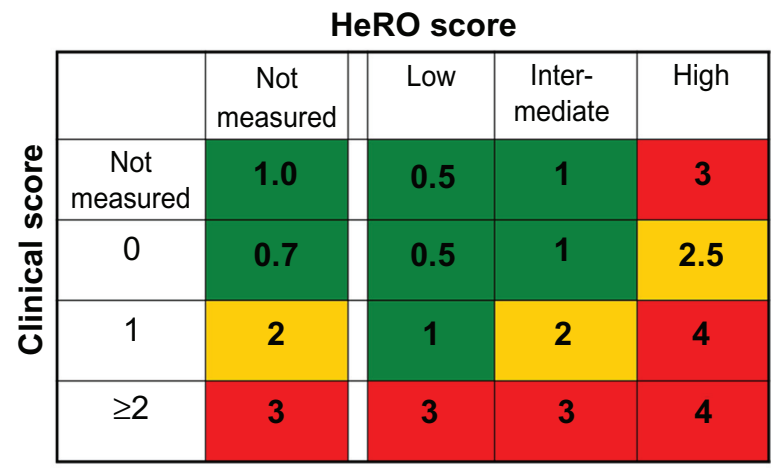

Figure 6 Score card for combining HeRO and clinical findings for diagnosis of sepsis. Notes: A clinical score was designed with I or 2 points given for sepsis-like signs. Combining HeRO and clinical score assessment was tested in 337 very low birthweight infants, and the numbers in boxes indicate the fold-increase in odds of the baby being diagnosed with sepsis in the subsequent 24-hour period. Combining $\mathrm{HeRO}$ score and clinical score gave better diagnostic accuracy for imminent sepsis. Abbreviation: HeRO, heart rate observation. 
count, and immature to total neutrophil ratio. ${ }^{26}$ The advantage of the HeRO score is that it is continuously displaying the risk of sepsis, whereas blood tests are only available if and when clinicians decide to order them either routinely or based on a change in a patient's clinical condition. In a study of preterm NICU patients, combining HeRO score and blood test results added independent information for sepsis diagnosis (increasing the area under the receiver-operating characteristic curve from 0.73 to 0.82$).{ }^{26} \mathrm{C}$-reactive protein testing is often used for decisions about antibiotic therapy in patients with sepsis-like symptoms. A recent study showed that, in NICU patients undergoing blood culture for suspected sepsis, concurrent C-reactive protein and HeRO score were not correlated, whereas some cytokine levels were associated with HeRO score elevation. ${ }^{27} \mathrm{C}$-reactive protein is often low at clinical presentation of sepsis, and rises 8-12 hours later. By the time $\mathrm{C}$-reactive protein is elevated, the systemic inflammatory response (and the HeRO score) may be subsiding, particularly if antibiotic therapy was initiated. However, following the $\mathrm{C}$-reactive protein levels may be useful for decisions about whether or not to continue antibiotic therapy beyond 24-48 hours, depending on blood culture results and other clinical parameters.

\section{Differential diagnosis of an increase in HeRO score}

While sepsis is the leading cause of an abrupt, large rise in HeRO score, other etiologies have been identified.
Patients with inflammatory conditions such as urinary tract infection and necrotizing enterocolitis, even in the absence of bacteremia, often have an acute increase in HeRO score. Acute respiratory decompensation, especially if associated with chest radiograph changes suggesting inflammation, can cause decreased HRV and/or decelerations contributing to a spike in the HeRO score. Apnea-associated heart rate decelerations, if very frequent, can also increase the HeRO score. However, it is important to note that many pathologic heart rate decelerations leading to HeRO score elevation are not related to pauses in breathing, as illustrated by the fact that infants on mechanical ventilation display decelerations during sepsis and during acute respiratory distress. There is evidence that pathogens or acute hypoxia can stimulate transient, repetitive heart rate decelerations, at least in part by vagus nerve activation. ${ }^{28,29}$

Surgery, including relatively minor procedures such as inguinal hernia repair, usually causes an acute increase in the HeRO score (Figure 7). Anesthetic agents, both inhaled and systemic, are known to suppress HRV. Typically, there is increased heart rate, sympathetic activation, and reduced cardiac vagal activity, and there is a dose-dependency to the effect. Other processes occurring intraoperatively or postoperatively which may impact HRV include hypovolemia, pain, or high doses of sedatives or analgesics. HRC (and HeRO score) typically normalize within 24 hours of surgery in absence of illness.

Acute brain injury can be associated with abnormal autonomic nervous system function, which may manifest as

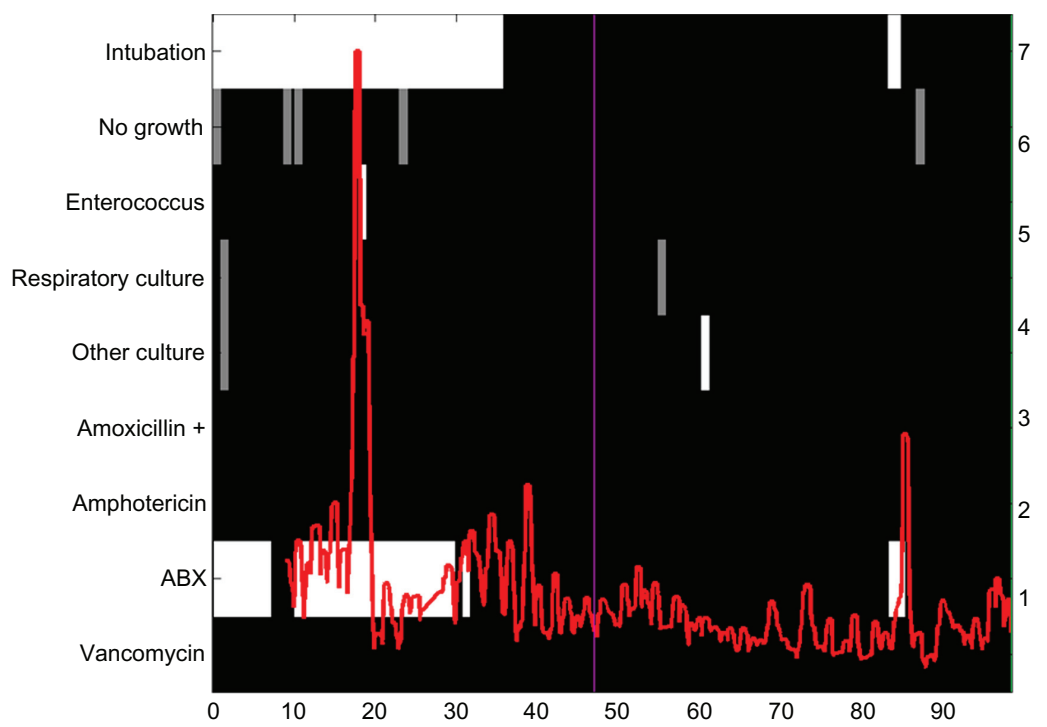

Figure 7 HeRO score over an entire stay in the neonatal intensive care unit showing two major spikes at the time of sepsis and surgery.

Notes: The HeRO score of a very low birth weight infant was monitored from day 9 of age through NICU discharge. The HeRO score (red line) ranged from $<1$ to 7 (right axis). On day 16, the patient had enterococcal septicemia, and on day 84 he had a minor surgical procedure. HeRO score was acutely elevated with both events. White and gray boxes indicate various clinical events, shown on the left.

Abbreviation: $\mathrm{HeRO}$, heart rate observation. 
abnormal HRC. In adults and children with traumatic brain injury, decreased HRV corresponds to increased intracranial pressure which is thought to result in compression of brain and brainstem centers involved in heart rate regulation. ${ }^{30,31}$ In focal or global ischemia-reperfusion injury such as stroke or asphyxia, HRV may also be depressed, ${ }^{32}$ and seizures also appear to impact HRV in some neonates. ${ }^{33}$ Preterm infants with brain ultrasound findings of intraventricular hemorrhage or periventricular leukomalacia have been reported to have decreased HRV. ${ }^{34}$ Correlation of high cumulative HeRO score and adverse neurodevelopmental outcomes (cognitive impairment and cerebral palsy at one year of age) was reported in a study of 65 preterm neonates. ${ }^{35}$ Our experience with monitoring HeRO scores in a large number of preterm infants over many years indicates that many of those with acute, severe intraventricular hemorrhage (IVH) have frequent large spikes in their HeRO score in the first weeks after birth, even in the absence of sepsis or acute respiratory compromise (Figure 8). IVH, particularly if it is associated with hydrocephalus or intraparenchymal damage, may directly cause abnormal autonomic nervous system activation and heart rate patterns. There is also evidence that cerebral injury in preterm neonates is associated with a prolonged systemic inflammatory response, ${ }^{36}$ which may indirectly lead to decreased HRV and decelerations accounting for a high HeRO score. These observations deserve further investigation to determine whether the HeRO monitor can be used as an adjunct test in assessing brain injury in NICU patients.

Certain types of cardiac disease would be predicted to alter HRV. Limited evidence in preterm infants suggests that presence of a patent ductus arteriosus is associated with decreased HRV, and indomethacin treatment for patent ductus arteriosus increases HRV. ${ }^{37,38}$ On the other hand, there is also evidence that treatment with prostaglandins to maintain ductal patency in patients with congenital heart disease increases HRV. ${ }^{38}$

Most drugs used in NICU patients have not been found to alter HeRO scores significantly, including vasopressor agents and inhaled beta adrenergic agonists. An exception is paralytic agents such as pancuronium, which cause an acute, transient decrease in HRV and increase in HeRO score. Anticholinergic agents such as atropine also dramatically dampen HRV, causing a transient increase in the score. This includes topical anticholinergic eye drops given prior to ophthalmologic examination, if they are systemically absorbed through the lacrimal duct.

Dexamethasone treatment consistently lowers the HeRO score in NICU patients. This may be due in part to suppression of inflammatory cytokines, which contribute to decreased HRV. Our work in a preclinical model has shown that dexamethasone also improves HRV independent of any inflammatory condition. ${ }^{39}$ Hydrocortisone, which is

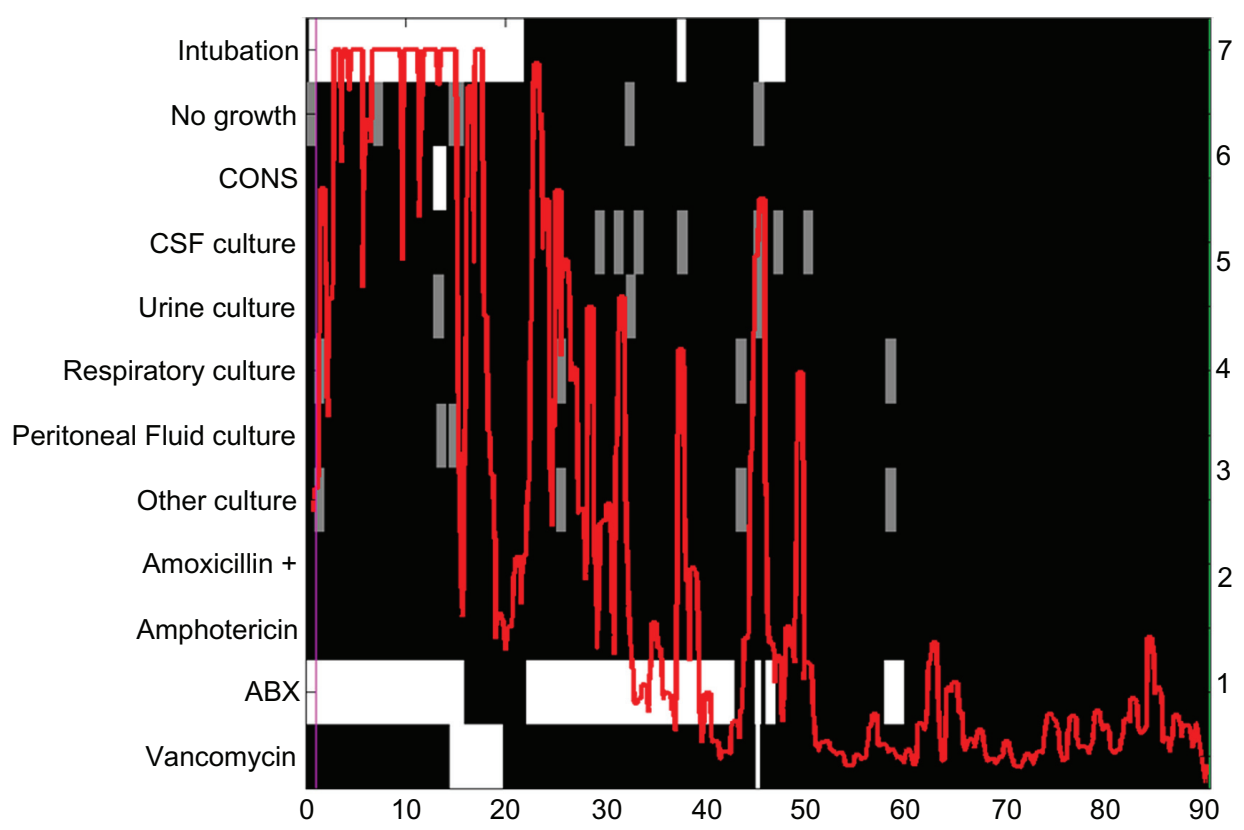

Figure $8 \mathrm{HeRO}$ score over an entire neonatal intensive care unit stay in an infant with acute brain injury.

Notes: The HeRO score of a 24-week infant was monitored from birth to discharge from the neonatal intensive care unit. He had a severe intraventricular hemorrhage day 2 leading to post-hemorrhagic hydrocephalus. He also had coagulase-negative staphylococcal septicemia on day I3, and multiple other cultures were negative. HeRO score was intermittently elevated for 7 weeks. White and gray boxes indicate the various clinical events, shown on left.

Abbreviations: HeRO, heart rate observation; CONS, coagulase negative staphylococcus. 
generally given in lower glucocorticoid-equivalent doses than dexamethasone and has additional mineralocorticoid activity, does not appear to have major effects on the HeRO score.

\section{"Spikiness" of HeRO score in sepsis}

Some patients manifest multiple peaks or "spikes" in the HeRO score prior to diagnosis of sepsis (Figure 9). We have considered several possible reasons for waxing and waning of abnormal HRC prior to clinical deterioration. Host defense mechanisms may be at play, with pathogen counts and cytokines in the blood increasing and decreasing in the preclinical phase as the immune system attempts to contain or eliminate the infection. Another possible explanation is that adrenergic and cholinergic receptors activated in infection and affecting HRC undergo downregulation with repeated stimulation. Our research in a mouse model indicates that pathogen-associated vagus nerve activation (leading to heart rate decelerations similar to those seen in septic neonates) undergoes desensitization, which can last hours or days. ${ }^{28}$ The advantage of the HeRO system may lie in its ability to intervene with antibiotics at one of the earlier (preclinical) spikes, thus preventing short-term and long-term complications associated with severe sepsis.

\section{Clinical dilemma: what to do with the "always spiky" sick baby?}

HeRO scores may display large peaks several times per week in very sick NICU patients, particularly those with severe lung or brain pathology (Figure 8). These babies are also at high risk for sepsis. The challenge for the clinician is to know how to interpret the HeRO score in a patient with a prior history of multiple spikes not associated with sepsis. In some cases, a patient-specific "stable baseline" can be identified. A large abrupt rise in HeRO over this baseline can then be used in conjunction with clinical signs or laboratory findings suggestive of infection to make decisions about obtaining cultures and starting antibiotics. Addition of biomarker testing such as C-reactive protein or a cytokine assay (if available) may improve the diagnostic utility of HeRO screening for making decisions about starting or stopping antibiotics in these complex and challenging patients. ${ }^{27}$

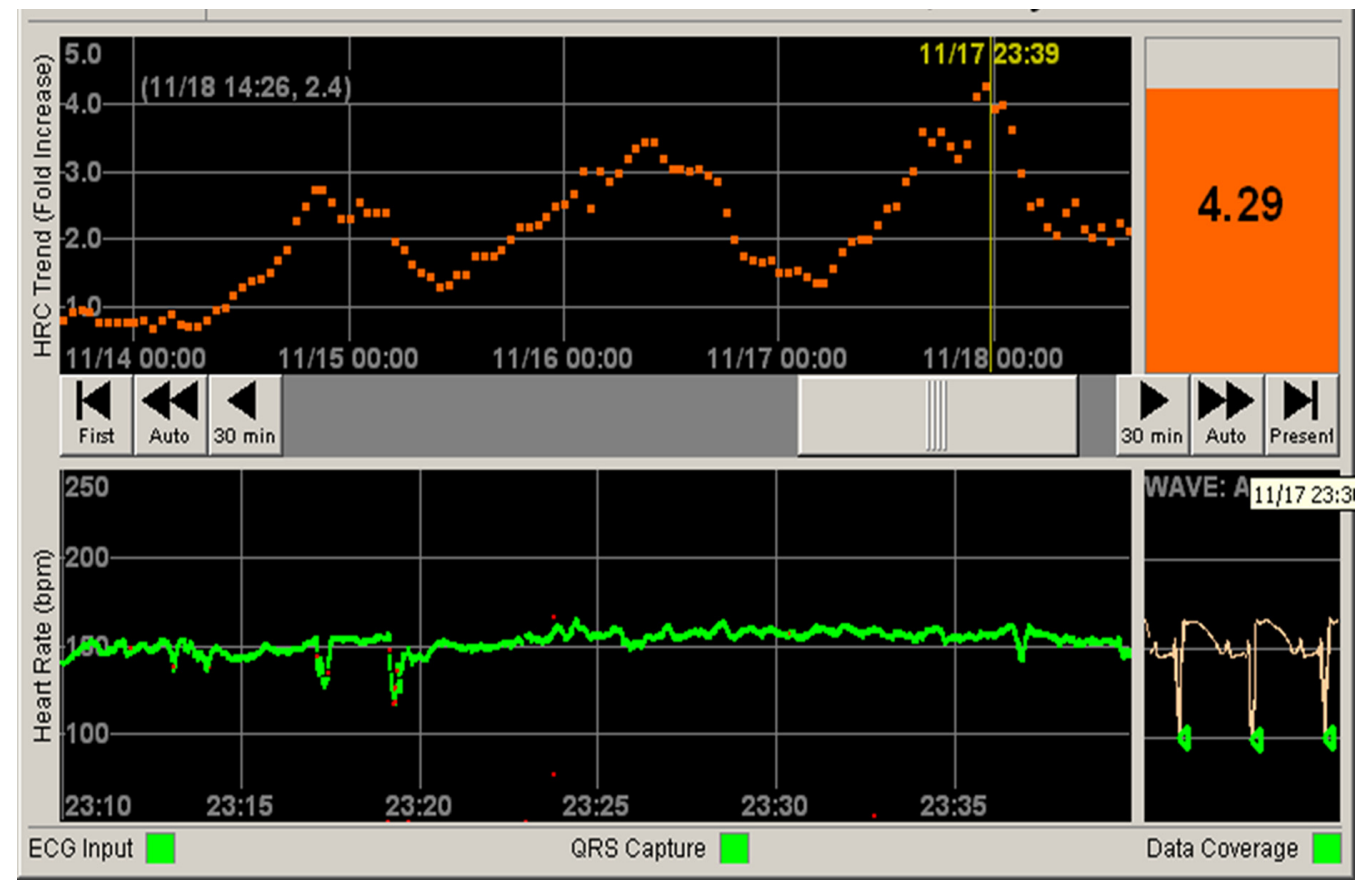

Figure 9 Multiple HeRO spikes prior to diagnosis of sepsis.

Notes: Some patients demonstrate waxing and waning of abnormal heart rate characteristics prior to clinical signs of sepsis and positive blood culture. In this case, the HeRO score acutely spiked from about I to progressively higher scores over a 4-day period. At the time indicated by the yellow line, the HeRO score was 4.29, and the associated 30-minute electrocardiogram shows decreased beat-to-beat variability with several superimposed decelerations. At that time, the patient had clinical signs of sepsis and a positive blood culture. Waxing and waning of abnormal heart rate characteristics in the days leading up to clinical diagnosis of septicemia may represent host defenses containing an infection for a time, or altered sensitivity of adrenergic or cholinergic receptors regulating heart rate. Acute increases in HeRO score in the days prior to clinical deterioration may represent opportunities for earlier diagnosis and treatment, leading to better outcomes.

Abbreviation: HeRO, heart rate observation. 


\section{HeRO scenarios}

The following scenarios are based on numerous actual cases and illustrate ways the HeRO score may be used in clinical practice.

\section{Scenario I}

An infant born at 25 weeks' gestation is 4 weeks old, "feeding and growing" in the intermediate care nursery. She develops a mild elevation in temperature and heart rate, thought to be due to overheating by the incubator. Her HeRO score has increased from 0.8 to 2.0 over the past 6 hours. She appears well and white blood cell count and differential are normal. Because of the rise in HeRO score, blood and urine cultures are obtained and antibiotics started. In 24 hours, Klebsiella pneumoniae is growing in blood and urine cultures. She receives a full course of antibiotics and does well.

\section{Scenario 2}

A 32-week gestation twin infant is 10 days old and tolerating advancing feeds. His HeRO score abruptly rises from $<1$ to $>3$ over a 4 -hour period, but he appears well. Cultures are obtained and antibiotics started. The HeRO score continues to rise, and 18 hours later the baby has clinical, radiographic, and laboratory evidence of necrotizing enterocolitis. $\mathrm{He}$ eventually recovers and is discharged home 7 weeks later on full enteral feeds.

\section{Scenario 3}

A 24-week gestation infant is 6 weeks old and was recently extubated. She has a history of apnea of prematurity, feeding intolerance, and multiple sepsis evaluations with negative cultures. The bedside nurse reports she is "not quite herself", with a modest increase in apnea spells and gastric residuals. The HeRO score remains $<1$. The decision is made to continue close observation and not perform a sepsis evaluation. The apnea and feeding intolerance improve and the HeRO score remains low over the next few days.

\section{Scenario 4}

A 23-week gestation infant has a grade 4 intraventricular hemorrhage detected on head ultrasound on day 2 and severe respiratory distress syndrome, leading to chronic lung disease requiring prolonged mechanical ventilation. Over the first 6 weeks after birth, his HeRO score routinely spikes from a baseline of 2 to 5 several times per week. Over this time period, he has had a number of blood cultures sent, several short courses of antibiotics with negative blood cultures, and one 14-day course of antibiotics for Klebsiella sepsis. Eventually, his condition improves, his HeRO score normalizes, and he is discharged home at 16 weeks of age.

\section{Future directions for HeRO and other computerized vital sign analysis}

A number of important questions remain to be investigated to optimize the clinical utility of the HeRO monitor. In the randomized HeRO clinical trial, there was no mandated intervention for an abrupt HeRO score elevation, and outcomes might be further improved with implementation of guidelines or protocols. Adding biomarker screening to HeRO monitoring might provide additional support for decisions about starting and stopping antibiotics. ${ }^{27}$ Beyond improving survival, it is critically important to assess whether HeRO monitoring improves neurodevelopmental outcomes. Additionally, it appears that elevated HeRO scores can be used to predict adverse neurodevelopmental outcomes, even after controlling for abnormalities on brain imaging, and this merits study in larger groups of patients. Finally, combining respiratory and heart rate pattern analysis for improved detection of sepsis and other life-threatening conditions may yield even greater improvement in clinical decision support and outcomes for NICU patients.

\section{Disclosure}

The authors report no conflicts of interest in this work.

\section{References}

1. Tyson JE, Parikh NA, Langer J, Green C, Higgins RD. Intensive care for extreme prematurity - moving beyond gestational age. NEngl JMed. 2008; 358:1672-1681

2. Stoll BJ, Hansen N. Infections in VLBW infants: studies from the NICHD Neonatal Research Network. Semin Perinatol. 2003;27:293-301.

3. Stoll BJ, Hansen NI, Adams-Chapman I, et al. Neurodevelopmental and growth impairment among extremely low-birth-weight infants with neonatal infection. JAMA. 2004;292:2357-2365.

4. Fairchild KD, O'Shea TM. Heart rate characteristics: physiomarkers for detection of late-onset neonatal sepsis. Clin Perinatol. 2010;37: 581-598.

5. Seely AJ, Green GC, Bravi A. Continuous multiorgan variability monitoring in critically ill patients - complexity science at the bedside. Conf Proc IEEE Eng Med Biol Soc. 2011;2011:5503-5506.

6. Lee H, Rusin CG, Lake DE, et al. A new algorithm for detecting central apnea in neonates. Physiol Meas. 2012;33:1-17.

7. Clark MT, Rusin CG, Hudson JL, et al. Breath-by-breath analysis of cardiorespiratory interaction for quantifying developmental maturity in premature infants. J Appl Physiol. 2012;112:859-867.

8. Buchman TG, Stein PK, Goldstein B. Heart rate variability in critical illness and critical care. Curr Opin Crit Care. 2002;8:311-315.

9. Kox M, Vrouwenvelder MQ, Pompe JC, van der Hoeven JG, Pickkers P, Hoedemaekers CW. The effects of brain injury on heart rate variability and the innate immune response in critically ill patients. J Neurotrauma. 2012;29:747-755. 
10. Brown AD, Barton DA, Lambert GW. Cardiovascular abnormalities in patients with major depressive disorder: autonomic mechanisms and implications for treatment. CNS Drugs. 2009;23:583-602.

11. Modanlou HD, Murata Y. Sinusoidal heart rate pattern: reappraisal of its definition and clinical significance. J Obstet Gynaecol Res. 2004;30: 169-180.

12. Ahmad S, Tejuja A, Newman KD, Zarychanski R, Seely AJ. Clinical review: a review and analysis of heart rate variability and the diagnosis and prognosis of infection. Crit Care. 2009;13:232.

13. Moorman JR, Lake DE, Griffin MP. Heart rate characteristics monitoring for neonatal sepsis. IEEE Trans Biomed Eng. 2006;53:126-132.

14. Jones SB, Romano FD. Myocardial beta adrenergic receptor coupling to adenylate cyclase during developing septic shock. Circ Shock. 1990;30:51-61.

15. Tracey KJ. Physiology and immunology of the cholinergic antiinflammatory pathway. J Clin Invest. 2007;117:289-296.

16. Borovikova LV, Ivanova S, Zhang M, et al. Vagus nerve stimulation attenuates the systemic inflammatory response to endotoxin. Nature. 2000;405:458-462.

17. Bravi A, Longtin A, Seely AJ. Review and classification of variability analysis techniques with clinical applications. Biomed Eng Online. 2011;10:90.

18. Griffin MP, Scollan DF, Moorman JR. The dynamic range of neonatal heart rate variability. J Cardiovasc Electrophysiol. 1994;5:112-124.

19. Griffin MP, Moorman JR. Toward the early diagnosis of neonatal sepsis and sepsis-like illness using novel heart rate analysis. Pediatrics. 2001;107:97-104.

20. Griffin MP, O’Shea TM, Bissonette EA, Harrell FE Jr, Lake DE, Moorman JR. Abnormal heart rate characteristics preceding neonatal sepsis and sepsis-like illness. Pediatr Res. 2003;53:920-926.

21. Richman JS, Moorman JR. Physiological time-series analysis using approximate entropy and sample entropy. Am J Physiol Heart Circ Physiol. 2000;278:H2039-H2049.

22. Kovatchev BP, Farhy LS, Cao H, Griffin MP, Lake DE, Moorman JR. Sample asymmetry analysis of heart rate characteristics with application to neonatal sepsis and systemic inflammatory response syndrome. Pediatr Res. 2003;54:892-898.

23. Godin PJ, Fleisher LA, Eidsath A, et al. Experimental human endotoxemia increases cardiac regularity: results from a prospective, randomized, crossover trial. Crit Care Med. 1996;24:1117-1124.

24. Griffin MP, Lake DE, Bissonette EA, Harrell FE Jr, O'Shea TM, Moorman JR. Heart rate characteristics: novel physiomarkers to predict neonatal infection and death. Pediatrics. 2005;115:1070-1074.

25. Griffin MP, Lake DE, O'Shea TM, Moorman JR. Heart rate characteristics and clinical signs in neonatal sepsis. Pediatr Res. 2007;61: $222-227$.
26. Griffin MP, Lake DE, Moorman JR. Heart rate characteristics and laboratory tests in neonatal sepsis. Pediatrics. 2005;115:937-941.

27. Raynor LL, Saucerman JJ, Akinola MO, Lake DE, Moorman JR, Fairchild KD. Cytokine screening identifies NICU patients with Gram-negative bacteremia. Pediatr Res. 2012;71:261-266.

28. Fairchild KD, Srinivasan V, Moorman JR, Gaykema RP, Goehler LE. Pathogen-induced heart rate changes associated with cholinergic nervous system activation. Am J Physiol Regul Integr Comp Physiol. 2011;300:R330-R339.

29. Lumbers ER, McCloskey DI, Potter EK, Courtice GP. Cardiac vagal action during hypoxia in adult and fetal sheep. JAuton Nerv Syst. 1986;16: 23-34.

30. Kox M, Vrouwenvelder MQ, Pompe JC, van der Hoeven JG, Pickkers P, Hoedemaekers CW. The effects of brain injury on heart rate variability and the innate immune response in critically ill patients. JNeurotrauma. 2012;29:747-755

31. Mowery NT, Norris PR, Riordan W, Jenkins JM, Williams AE, Morris JA Jr. Cardiac uncoupling and heart rate variability are associated with intracranial hypertension and mortality: a study of 145 trauma patients with continuous monitoring. J Trauma. 2008;65:621-627.

32. Aliefendioglu D, Dogru T, Albayrak M, Dibekmisirlioglu E, Sanli C. Heart rate variability in neonates with hypoxic ischemic encephalopathy. Indian J Pediatr. February 23, 2012. [Epub ahead of print.]

33. Malarvili MB, Mesbah M. Newborn seizure detection based on heart rate variability. IEEE Trans Biomed Eng. 2009;56:2594-2603.

34. Hanna BD, Nelson MN, White-Traut RC, et al. Heart rate variability in preterm brain-injured and very-low-birth-weight infants. Biol Neonate. 2000;77:147-155

35. Addison K, Griffin MP, Moorman JR, Lake DE, O'Shea TM. Heart rate characteristics and neurodevelopmental outcome in very low birth weight infants. J Perinatol. 2009;29:750-756.

36. Leviton A, Kuban K, O'Shea TM, et al. The relationship between early concentrations of 25 blood proteins and cerebral white matter injury in preterm newborns: the ELGAN study. J Pediatr. 2011;158:897-903.

37. van Ravenswaaij-Arts CM, Hopman JC, Kollee LA, van Amen JP, Stoelinga GB, van Geijn HP. The influence of respiratory distress syndrome on heart rate variability in very preterm infants. Early Hum Dev. 1991;27:207-221.

38. Prietsch V, Maier R, Schmitz L, Obladen M. Long-term variability of heart rate increases with successful closure of patent ductus arteriosus in preterm infants. Biol Neonate. 1992;61:142-149.

39. Fairchild KD, Saucerman JJ, Raynor LL, et al. Endotoxin depresses heart rate variability in mice: cytokine and steroid effects. Am J Physiol Regul Integr Comp Physiol. 2009;297:R1019-R1027.
Research and Reports in Neonatology

\section{Publish your work in this journal}

Research and Reports in Neonatology is an international, peer-reviewed, open access journal publishing original research, reports, editorials, reviews and commentaries on neonatal health. The manuscript management system is completely online and includes a very quick and fair
Dovepress

peer-review system. Visit http://www.dovepress.com/testimonials.php to read real quotes from published authors. 